\title{
Singularities in Fully Developed Turbulence
}

\author{
Bhimsen K. Shivamoggi \\ University of Central Florida \\ Orlando, FL 32816-1364, USA
}

\begin{abstract}
Phenomenological arguments are used to explore finite-time singularity (FTS) development in different physical fully-developed turbulence (FDT) situations. Effects of spatial intermittency and fluid compressibility in three-dimensional (3D) FDT and the role of the divorticity amplification mechanism in two-dimensional (2D) FDT and quasi-geostrophic FDT and the advection-diffusion mechanism in magnetohydrodynamic turbulence are considered to provide physical insights into the FTS development in variant cascade physics situations. The quasi-geostrophic FDT results connect with the 2D FDT results in the barotropic limit while they connect with 3D FDT results in the baroclinic limit and hence apparently provide a bridge between 2D and 3D.
\end{abstract}




\section{Introduction}

The existence of strongly localized features like vorticity sheets in the small-scale structure of three-dimensional (3D) fully-developed turbulence (FDT) suggests the development of singularities in the flow variables ${ }^{1}$ (Gibbon [1]). On the mathematical side, the 3D Euler equations $^{2}$ may be viewed as a quadratic evolution equation for vorticity $\boldsymbol{\omega}$ which, in principle, allows some solutions to evolve from smooth initial data to a singularity in finite time (Constantin [3]), so this possibility (without a rigorous proof yet) is of interest to mathematicians in the context of global existence of solutions. ${ }^{3}$ It is generally believed that an understanding of this process ${ }^{4}$ is crucial to the development of a viable theory of turbulence (Constantin [3]). A rigorous result due to Beale et al. [5] requires the magnitude of the vorticity $\boldsymbol{\omega}$ to become infinite to allow the occurrence of a finite-time singularity (FTS $)^{5}$. More specifically, this result states that a singularity in a flow variable develops as $t \rightarrow t^{*}$, only if

$$
\int_{0}^{t}\|\omega\|_{\infty}(\tau) d \tau \rightarrow \infty, \text { as } t \rightarrow t^{*}
$$

This result implies

$$
\|\omega\|_{\infty}(t) \sim \frac{1}{\left(t^{*}-t\right)^{\beta}}, \beta \geq 1 .
$$

Here, the $L^{\infty}$ norm $\|\omega\|_{\infty} \equiv \max _{\boldsymbol{x} \in \mathbb{R}^{3}}\|\omega(\boldsymbol{x})\|$. Another result due to Constantin et al. [8], [9] postulates that the curvature of vortex lines should tend to infinity in order that the magnitude of the vorticity $\boldsymbol{\omega}$ becomes infinity. Ruban et al. [10] pointed out that the vortex line curvature blow-up is actually more critical to the FTS development than the vorticity magnitude blow-up. ${ }^{6}$

Numerical investigations of 3D FDT (see Gibbon [1] for a detailed summary) have suggested but failed to provide a conclusive evidence that ideal-flow solutions, starting from regular initial conditions, will spontaneously develop a singularity in finite time (for example, Brachet et al. [12]). Mathematically, it is not yet resolved if the Euler equations are guaranteed to possess solutions that remain smooth, for all $t$, if the initial data are smooth. The existing proofs establish either existence of smooth solutions, which is local in $t$, or weak solutions, for all $t$, whose smoothness is not guaranteed. The outstanding open issue therefore is (Majda and Bertozzi [13]), "Are there smooth solutions with finite energy of the 3D Euler equations that develop singularities in a finite time?"

\footnotetext{
${ }^{1}$ The mechanism of vorticity stretching and amplification typically involves the generation of small-scale structures (example: the Burgers vortex).

${ }^{2}$ For a recent discussion of 3D Euler equations, see Weidemann [2].

${ }^{3}$ Indeed, Leray [4] originally recognized the possibility of self-similar collapse towards a singularity as $t \rightarrow t^{*}$, say, with length scales decreasing in proportion to $\left(t^{*}-t\right)^{1 / 2}$ and the associated vorticity field $\boldsymbol{\omega}(\mathbf{x}, t)$ scaling like $\left(t^{*}-t\right)^{-1}$.

${ }^{4}$ One of the issues in this regard is the connection, if any, between the energy transfer to small scales and dissipative anomaly associated with the time-asymmetry property in FDT and the finite-time singularity.

${ }^{5} \boldsymbol{\omega}$ amplifies when it roughly aligns with the linear eigenspace associated with the positive intermediate eigenvalue of the local flow-strain matrix (Majda [6]) while the latter is found to be associated with the time-asymmetry property in FDT (Jucha et al. [7]).

${ }^{6}$ The curvature of a vortex sheet was shown to undergo a FTS development (Moore [11]).
} 
Phenomenological considerations, which predict development of FTS in FDT, are believed to over-estimate the nonlinear effects (Frisch [14]) because nonlinearity depletion mechanisms via local alignment of vorticity and the consequent coherent structure generation seem to be operational (Constantin [3], Frisch [14]) and enhanced coherence of the vorticity field makes the conditions for the singularity development more stringent. Nonetheless, phenomenological arguments can provide a useful description of the dynamics of the FTS development, and and in particular, the asymptotic behavior of the field variable near the singularity, if one exists. Therefore, here we propose to use phenomenological arguments to explore the FTS development in different physical FDT situations because, from a qualitative point of view, such considerations do seem to be able to provide useful physical insights into this process in variant cascade physics situations as well as interesting connections between the results pertaining to the latter cases.

\section{3D Incompressible FDT}

Vortex stretching and amplification is believed to constitute the physical mechanism underlying the energy cascade in 3D FDT. So, the vorticity $\boldsymbol{\omega} \equiv \nabla \times \mathbf{v}$ is the appropriate physical variable to characterize the dynamics in question. An estimate for the energy dissipation rate $\varepsilon$ in $3 \mathrm{D}$ FDT is given by,

$$
\varepsilon \sim \nu \frac{v^{2}}{\eta^{2}}
$$

$v$ being the velocity increment over the Kolmogorov microscale $\eta$ and $\nu$ being the kinematic viscosity. On using the Kolmogorov scaling,

$$
v \sim \varepsilon^{1 / 3} \eta^{1 / 3}
$$

(2) gives the well-known result,

$$
\eta \sim \frac{\nu^{3 / 4}}{\varepsilon^{1 / 4}}
$$

On using (4), the vorticity evolution equation,

$$
\frac{d \boldsymbol{\omega}}{d t}=(\boldsymbol{\omega} \cdot \nabla) \mathbf{v}+\nu \nabla^{2} \boldsymbol{\omega}
$$

leads to the vorticity growth rate estimate, ${ }^{7}$

$$
\frac{d \omega}{d t} \sim \nu \frac{\omega}{\eta^{2}} \sim \frac{\varepsilon^{1 / 2}}{\nu^{1 / 2}} \omega
$$

\footnotetext{
${ }^{7}$ If one uses the vorticity stretching term in (5), (instead of the viscous term as in (6)), we obtain the following estimate for the vorticity growth rate

and on using (3) and (4),

$$
\frac{d \omega}{d t} \sim \frac{\omega v}{\eta}
$$$$
\frac{d \omega}{d t} \sim \frac{\varepsilon^{1 / 3}}{\eta^{2 / 3}} \omega \sim \frac{\varepsilon^{1 / 2}}{\nu^{1 / 2}} \omega
$$

in agreement with (6). Thus, the viscous dissipation and vortex stretching terms in equation (5) lead to equivalent estimates.
} 
Invoking the dissipative anomaly, in 3D FDT,

$$
\varepsilon \sim \nu \omega^{2} \sim \text { const }
$$

(6) leads to

$$
\frac{d \omega}{d t} \sim \omega^{2}
$$

and hence the well-known result,

$$
\omega \sim \frac{1}{t+c}
$$

suggesting a FTS in 3D FDT, in qualitative agreement with Leray's [4] self-similar collapse result (see footnote 3). Here, $c$ is an arbitrary constant. There were some indications of this kind of behavior in numerical experiments (Pumir and Siggia [15], Pelz [16] and Grauer et al. $[17])^{8}$.

\section{Effects of Spatial Intermittency in 3D FDT}

Spatial intermittency effects associated with the spatial spottiness of the turbulent activity become more pronounced at small scales. So, one may surmise spatial intermittency to have a significant effect on the FTS development. One may then incorporate spatial intermittency effects, following Mandelbrot [19], via the fractal nature of strongly convoluted dissipative structures where the turbulent activity is concentrated. In a first approximation, the dissipative structures may be approximated by a homogeneous fractal ${ }^{9}$ with a non-integer Hausdorff dimension $D_{0}$ (Frisch et al. [20]).

Assuming the scaling behavior ${ }^{10}$,

$$
v \sim \eta^{\alpha}
$$

we have,

$$
\varepsilon \sim \eta^{3 \alpha-1} .
$$

Using (11), (4) gives (Paladin and Vulpiani [21], Sreenivasan and Meneveau [22]),

$$
\eta \sim \nu^{\frac{1}{1+\alpha}}
$$

The homogeneous fractal model for the 3D FDT (Frisch et al. [20]) gives

$$
\alpha=\frac{1}{3}\left(D_{0}-2\right) \text {. }
$$

\footnotetext{
${ }^{8}$ The deduction of correct FTS estimates provides an operational validation for using steady state estimates (the scaling laws) in the initial-value problem. The apparent mathematical inconsistency in this process may be remedied by assuming the existence of disparate time scales in the problem. The scaling laws may be assumed to build up rapidly and attain their asymptotic forms before the FTS development materializes. This situation is similar to the Bogliubov hypothesis in kinetic theory of gases, which introduces the time arrow and hence irrversibility into the system (the H-Theorem). It therefore appears plausible that the use of the asymptotic forms in the FTS development process may indeed be legitimized by the dissipative anormaly and the consequent introduction of irreversibility (even in the inviscid limit) into the FDT system (Falkovich et al. [18]). Geometry is believed to play an important role in this development (Grisha Falkovich, private communication.)

${ }^{9}$ In a homogeneous fractal model (also called the $\beta$-model) for the dissipative structures, the energy flux is assumed to be transferred to only a fixed function $\beta$ of the eddies downstream in the cascade (Frisch et al. [20]).

${ }^{10}$ The non-differentiability of the velocity field implies that the Hölder scaling exponent $\alpha$ satisfies the condition $\alpha \leq 1 / 3$ (as confirmed by (13) below).
} 
Using (13), (12) gives

$$
\eta \sim \nu^{\frac{3}{D D_{0}+1}} .
$$

Using (14), (5) leads to the vorticity growth rate estimate,

$$
\frac{d \omega}{d t} \sim \nu \frac{\omega}{\eta^{2}} \sim \nu^{\frac{D_{0}-5}{D_{0}+1}} \omega .
$$

Using (7), (15) becomes

$$
\frac{d \omega}{d t} \sim \omega^{\frac{11-D_{0}}{D_{0}+1}}
$$

from which,

$$
\omega \sim(t+c)^{-1+\frac{3}{2}\left(\frac{D_{0}-3}{D_{0}-5}\right) .}
$$

The weakening of the FTS due to spatially intermittency $\left(D_{0}<3\right)$, indicated by (16), reflects a nonlinearily depletion activity occurring in the latter case via generation of coherent structures ${ }^{11}$ and the consequent enhanced coherence of the vorticity field, as conjectured by Frisch [14].

\section{Effects of Compressibility}

Compressibility effects on FDT are of great importance in modern technological as well as astrophysical flows (Shivamoggi [24] and other references given thereof). There is an intuitive belief that vortices tend to become more resilient and stretch stronger in a compressible fluid. It is therefore pertinent to explore the effect of fluid compressibility on the FTS development.

For the compressible case, an estimate for the kinetic energy dissipation rate $\hat{\varepsilon}$ is given by

$$
\hat{\varepsilon} \sim \frac{\rho v^{3}}{\hat{\eta}} \sim \mu \frac{v^{2}}{\hat{\eta}^{2}}
$$

from which, we obtain

$$
\left.\begin{array}{l}
\hat{\eta} \sim \frac{\mu}{\rho v} \\
v \sim\left(\frac{\hat{\varepsilon} \hat{\eta}}{\rho}\right)^{1 / 3}
\end{array}\right\}
$$

and hence (Shivamoggi [24]), the Kolmogorov microscale $\hat{\eta}$ for compressible FDT is given by

$$
\hat{\eta} \sim\left(\frac{\mu^{3}}{\rho^{2} \hat{\varepsilon}}\right)^{1 / 4} .
$$

The vorticity growth rate estimate, ${ }^{12}$

\footnotetext{
${ }^{11}$ Mailybaev [23] suggests, by considering a shell model for FDT, that the coherent structure generation may also be induced by the FTS.

${ }^{12}$ The vorticity evolution equation for a compressible, barotropic fluid is

$$
\frac{d \boldsymbol{\omega}}{d t}+\boldsymbol{\omega}(\nabla \cdot \mathbf{v})=(\boldsymbol{\omega} \cdot \nabla) \mathbf{v}+\mu\left[\frac{1}{\rho} \nabla^{2} \boldsymbol{\omega}-\frac{1}{\rho^{2}} \nabla \rho \times\left\{\nabla^{2} \mathbf{v}+\frac{1}{3} \nabla(\nabla \cdot \mathbf{v})\right\}\right] .
$$
}




$$
\rho \frac{d \omega}{d t} \sim \mu \frac{\omega}{\hat{\eta}^{2}}
$$

on using (20), becomes

$$
\frac{d \omega}{d t} \sim \frac{\hat{\varepsilon}^{1 / 2}}{\mu^{1 / 2}} \omega
$$

Noting the dissipative anomaly for the compressible case (Shivamoggi [25]),

$$
\hat{\varepsilon} \sim \mu \omega^{2} \sim \text { const }
$$

(22) becomes

$$
\frac{d \omega}{d t} \sim \omega^{2}
$$

and hence

$$
\omega \sim \frac{1}{t+c}
$$

as in the incompressible case. The apparent absence of a compressibility correction to (9) is probably due to the fact that compressibility effects are not length-scale dependent and materialize equally at all length scales unlike viscous effects.

On the other hand, in the ultimate compressibility limit, the dissipative structures become shock waves, which may be associated with the development of singularities in the flow variables in a compressible FDT (Mailybaev [26]). In this limit, we have (Shivamoggi [24]) ${ }^{13}$,

$$
\left.\begin{array}{l}
\rho \sim \hat{\eta} \\
v \sim \text { const } \\
\omega \sim \hat{\eta}^{-1}
\end{array}\right\} .
$$

Using (26a, b, c), (21) leads to

$$
\frac{d \omega}{d t} \sim\left(\mu \omega^{2}\right) \omega^{2}
$$

and using (23), (27) leads to

$$
\frac{d \omega}{d t} \sim \omega^{2}
$$

in agreement with (24) even in this limit.

\footnotetext{
${ }^{13}$ It may be noted that $(26 \mathrm{a}, \mathrm{b})$ lead to the following scaling behavior for the kinetic energy per unit mass,$$
\rho v^{2} \sim \hat{\eta}
$$

which, in turn, leads to the Kadomtsev-Petviashvili [27] spectral law for compressible FDT,

$$
E(k) \sim k^{-2}
$$

$E$ being the kinetic energy spectral density and $k$ being the wave number.
} 


\section{Enstrophy Cascade in 2D FDT}

As a further example of the effect of the underlying cascade physics on the FTS development, let us consider the enstrophy cascade in 2D FDT. 2D FDT is relevant to large-scale atmospheric and oceanic flows and radically differs from 3D FDT in that it does not have the vortex stretching mechanism operational in 3D ${ }^{14}$ (see Tabeling [28] for a recent review) 15 .

Noting that divorticity (Kida [38]) amplification constitutes the physical mechanism underlying the enstrophy cascade in 2D FDT (Kuznetsov et al. [39]) the divorticity b,

$$
\mathbf{b} \equiv \nabla \times \omega
$$

appears to be the appropriate physical variable to characterize the dynamics in question (Shivamoggi et al. [40]).

The divorticity evolution equation,

$$
\frac{d \mathbf{b}}{d t}=(\mathbf{b} \cdot \nabla) \mathbf{v}+\nu \nabla^{2} \mathbf{b}
$$

on noting that the Kraichnan microscale $\zeta$ (which is the 2D FDT counterpart of the Kolmogorov microscale for 3D FDT) is given by (Shivamoggi [41]),

$$
\zeta \sim \frac{\nu^{1 / 2}}{\tau^{1 / 6}}
$$

leads to the divorticity growth rate estimate,

$$
\frac{d b}{d t} \sim \nu \frac{b}{\zeta^{2}} \sim \tau^{1 / 3} b .
$$

Here, $\tau$ is the enstrophy dissipation rate. (32) further leads to

$$
b \sim e^{\tau^{1 / 3} t}
$$

\footnotetext{
${ }^{14}$ The vorticity evolution equation in $2 \mathrm{D}$,

$$
\frac{d \boldsymbol{\omega}}{d t}=\mathbf{0}
$$

implies the absence of the vortex stretching mechanism in $2 \mathrm{D}$ and leads to the Lagrange invariant,$$
\boldsymbol{\omega}=\text { const. }
$$

This invariant introduces a strong restriction on the dynamics underlying $2 \mathrm{D}$.

${ }^{15}$ The scaling laws in 2D enstrophy range have been shown (Eyink [29]) to correspond to the inviscid limit of the Leray solutions of 2D Navier-Stokes equations and have some essential differences with the scaling laws in 3D inertial range,

* non-locality of the nonlinear interactions in the spectral space;

* non-operationality of the usual nonlinearity-sustained cascade mechanism (Lopes Filho et al. [30]).

However, the presence of spatial intermittency, which may be due either to coherent structures or (Paret et al. [31], Schneider and Forge [32]) or to contamination from 3D effects (as in any real flow situation) restores,

* the spectral localness of the nonlinear interactions (McWilliams [33], She et al. [34]);

* the nonlinearity-sustained enstrophy cascade mechanism (Lopes Filho et al. [30], Shivamoggi [35]);

and hence produces a $3 \mathrm{D}$-like situation in the $2 \mathrm{D}$ enstrophy range. The radical effect of intermittency on the $2 \mathrm{D}$ enstrophy range dynamics also occurs in the relative particle dispersion problem (RPD) - even a slight intermittency turns out to make the $\mathrm{RPD}$ to go from an exponential growth type to an algebraic growth type (Shivamoggi [36]) observed in laboratory experiments (Jullien [37]).
} 
confirming the well known absence of a FTS in 2D FDT (Rose and Sulem [42]) consequent to the global-in-time regular behavior of 2D Navier-Stokes solutions evolving from smooth initial data in the inviscid limit.

\section{Enstrophy Cascade in Quasi-geostrophic FDT}

As indicated by the development in Section 5, the Lagrange invariance of vorticity introduces a strong restriction on the dynamics underlying $2 \mathrm{D}$ flows. It is of interest to consider the effect of a violation of this property on the FTS development. As an example, consider the enstrophy cascade in quasi-geostrophic ${ }^{16} \mathrm{FDT}$, which is relevant to large-scale oceanic flows with the inclusion of the Coriolis force and free-surface effects. The possibility of a FTS in the quasi-geostrophic model and its variants has been numerically discussed by Hoyer and Sadourny [44], Constantin et al. [45], Ohkitani and Yamada [46], Scott and Dritschel [47].

For this case, the Kraichnan microscale $\zeta$ is given by (see Appendix),

$$
\zeta \sim \begin{cases}\frac{\nu^{1 / 2}}{\tau^{1 / 6}} & , \zeta \ll R \\ \frac{\nu^{3 / 4}}{R^{1 / 2} \tau^{1 / 4}} & , \zeta \gg R .\end{cases}
$$

Here, $R$ is the Rossby deformation radius $R \equiv \sqrt{g H} / f, f$ is the local Coriolis parameter, $H$ is the depth of the ocean (taken to be constant), and $g$ is the acceleration due to gravity. We are using the simplest mathematical model of large-scale, nearly horizontal oceanic motion incorporating the force of gravity and the Coriolis force due to the Earth's rotation, which is the one-layer homogeneous ocean with a uniform depth and a spherical free surface. (34a), which corresponds to the barotropic regime, is the same as the 2D FDT result (31), while (34b), which corresponds to the baroclinic regime, is very similar to the 3D FDT result (4). This implies that the quasi-geostrophic FDT results have the potential to connect, in some sense, with the 2D FDT results in the barotropic limit and with the 3D FDT results in the baroclinic limit. This is confirmed in the following.

Using (34a, b), equation (30) leads to the divorticity growth rate estimate,

$$
\frac{d b}{d t} \sim \begin{cases}\tau^{1 / 3} b & , \zeta \ll R \\ \frac{R \tau^{1 / 2}}{\nu^{1 / 2}} b & , \zeta \gg R .\end{cases}
$$

Invoking the dissipative anomaly in quasi-2D FDT,

$$
\tau \sim \nu b^{2} \sim \text { const }
$$

$(35 \mathrm{a}, \mathrm{b})$ leads to

$$
\frac{d b}{d t} \sim \begin{cases}\tau^{1 / 3} b & , \zeta \ll R \\ R b^{2} & , \zeta \gg R\end{cases}
$$

\footnotetext{
${ }^{16}$ Quasi-geostrophic dynamics refers to the nonlinear dynamics governed by the first-order departure from the linear geostrophic balance between the Coriolis force and pressure gradient transverse to the rotation axis of a rapidly rotating fluid. Quasi-geostrophic FDT refers to randomly varying flow states of fluids that lie close to a state of geostrophic and hydrostatic balance (Charney [43]).
} 
and hence,

$$
b \sim \begin{cases}e^{\tau^{1 / 3} t} & , \zeta \ll R \\ \frac{1}{R t+c} & , \zeta \gg R\end{cases}
$$

suggesting, in the baroclinic limit, a FTS of the same type as that for vorticity in 3D FDT, namely, (9). Physically, this is due to the enhanced vortex stretching in this limit produced by the deformed free surface in the quasi-geostrophic dynamics.

Thus, the quasi-geostrophic FDT results connect with the 2D FDT results, as to be expected, in the barotropic limit while they connect with the 3D FDT results in the baroclinic limit (on doing the necessary interchange of the appropriate field variable divorticity in the quasi-2D case with vorticity in the 3D case). Contingent on the latter qualification, the quasi-geostrophic FDT seems to provide some kind of link between the 2D FDT and the 3D FDT.

\section{Magnetohydrodynamic Turbulence}

Magnetohydrodynamic (MHD) FDT is relevant to plasmas in astrophysical systems as well as fusion reactors (see Carbone and Pouquet [48] for a recent review). The advectiondiffusion mechanism controls the statistical properties of magnetic field ${ }^{17}$ in MHD FDT (especially if the applied magnetic field is weak, Bershadskii and Sreenivasan [51]). The MHD FDT (Shivamoggi [52]) therefore presents a convenient framework to explore the role of the advection-diffusion mechanism in the FTS development. Here, we adopt the Iroshnikov [53] - Kraichnan [54] (IK) phenomenology for illustration, and the full details (as well as controversies) of the MHD FDT cascade physics are outside the scope of the present discussion.

The energy dissipation rate in the IK model for MHD FDT is given by

$$
\varepsilon \sim \frac{v^{4}}{\tilde{\eta} C_{A}} \sim \eta_{m} \frac{v^{2}}{\tilde{\eta}^{2}}
$$

where $\eta_{m}$ is the magnetic resistivity, $C_{A}$ is the velocity of Alfvén waves in the magnetic field of the large-scale eddies, and $\tilde{\eta}$ is the Kolmogorov microscale for MHD FDT. We obtain from $(39)$,

$$
\left.\begin{array}{l}
\tilde{\eta} \sim \frac{\eta_{m} C_{A}}{v^{2}} \\
v \sim \varepsilon^{1 / 4} C_{A}^{1 / 4} \tilde{\eta}^{1 / 4}
\end{array}\right\} .
$$

Combining (40a, b), we obtain

$$
\tilde{\eta} \sim\left(\frac{\eta_{m}^{2} C_{A}}{\varepsilon}\right)^{1 / 3}
$$

\footnotetext{
${ }^{17}$ Advective stretching of magnetic field lines leads to amplification of magnetic field (Batchelor [49]) while magnetic field lines that have been highly stretched typically experience stronger Ohmic dissipation (Kida et al. [50]).
} 
The current density $J$ growth rate estimate, ${ }^{18}$

$$
\frac{d J}{d t} \sim \eta_{m} \frac{J}{\tilde{\eta}^{2}}
$$

on using (41), becomes

$$
\frac{d J}{d t} \sim \frac{\varepsilon^{2 / 3}}{C_{A}^{2 / 3} \eta_{m}^{1 / 3}} J .
$$

Noting the dissipative anomaly for MHD FDT (Shivamoggi [52]),

$$
\varepsilon \sim \eta_{m} J^{2} \sim \text { const }
$$

(43) becomes

$$
\frac{d J}{d t} \sim \frac{\varepsilon^{1 / 3}}{C_{A}^{2 / 3}} J^{5 / 3}
$$

(45) leads to

$$
J \sim \frac{1}{(t+c)^{3 / 2}}
$$

showing a strengthening of the FTS in the IK phenomenology, which is plausible because the MHD flows are known to be more dissipative than their hydrodynamic counterparts (Orszag and Tang [55]).

\section{Discussion}

In this paper phenomenological arguments have been used to provide some physical insights into the FTS development in different physical FDT situations. Particular attention is paid to the role played by the cascade physics underlying the FTS development. Such diverse aspects as the effects of spatial intermittency and fluid compressibility in 3D FDT and the role of the divorticity amplification mechanism in 2D FDT and quasi-2D quasigeostrophic FDT and the advection-diffusion mechanism in MHD turbulence are considered to gain better physical understanding of the FTS development in variant cascade-physics situations as well as interesting connections between the results pertaining to the latter cases. The quasi-geostrophic FDT results connect with the 2D FDT results, as to be expected, in the barotropic limit while they connect with the 3D FDT results in the baroclinic limit (on doing the necessary interchange of the appropriate field variable divorticity in the quasi-2D case with vorticity in the $3 \mathrm{D}$ case). In this sense, the quasi-geostrophic FDT appears to provide a kind of bridge between the 2D FDT and the 3D FDT.

\footnotetext{
${ }^{18}$ The current density evolution equation is

$$
\frac{\partial \mathbf{J}}{\partial t}-\nabla \times[\nabla \times(\mathbf{v} \times \mathbf{B})]=\eta_{m} \nabla^{2} \mathbf{J}
$$
}

B being the magnetic field. 


\section{Appendix}

Noting that the potential enstrophy for quasi-geostrophic flows in the Charney [43] model ${ }^{19}$ is given by

$$
U \sim \frac{\phi^{2}}{\ell^{2}}\left(\frac{1}{\ell^{2}}+\frac{1}{R^{2}}\right)
$$

we have for the enstrophy transfer rate estimate,

$$
\tau \sim \begin{cases}\frac{\phi^{3}}{\ell^{6}} & , \ell \ll R \\ \frac{\phi^{3}}{R^{2} \ell^{4}} & , \ell \gg R\end{cases}
$$

$\phi$ being the stream function.

Assuming that the enstrophy transfer rate is constant in the inertial range, we obtain from (A.2a, b),

$$
\phi \sim \begin{cases}\tau^{1 / 3} \ell^{2} & , \ell \ll R \\ \tau^{1 / 3} R^{2 / 3} \ell^{4 / 3} & , \ell \gg R .\end{cases}
$$

On the other hand, using (A.1), the enstrophy dissipation rate is given by

$$
\tau \sim\left\{\begin{array}{cc}
\nu \frac{\phi^{2}}{\zeta^{6}} & , \zeta \ll R \\
\nu \frac{\phi^{2}}{R^{2} \zeta^{4}} & , \zeta \gg R
\end{array}\right.
$$

$\zeta$ being the Kraichnan microscale.

Using (A.3a, b), (A.4a, b) becomes

$$
\tau \sim \begin{cases}\frac{\nu \tau^{2 / 3}}{\zeta^{2}} & , \zeta \ll R \\ \frac{\nu \tau^{2 / 3}}{R^{2 / 3} \zeta^{4 / 3}} & , \zeta \gg R\end{cases}
$$

from which,

$$
\zeta \sim \begin{cases}\frac{\nu^{1 / 2}}{\tau^{1 / 6}} & , \zeta \ll R \\ \frac{\nu^{3 / 4}}{R^{1 / 2} \tau^{1 / 4}} & , \zeta \gg R\end{cases}
$$

as mentioned in $(34 \mathrm{a}, \mathrm{b})$.

\footnotetext{
${ }^{19}$ The vorticity evolution equation in quasi-geostrophic flows is (Charney [43])

$$
\frac{\partial}{\partial t}\left(\omega-\frac{\phi}{R^{2}}\right)+(\mathbf{v} \cdot \nabla) \omega=\nu \nabla^{2} \omega
$$
}

where $\omega=\nabla^{2} \phi, \phi$ being the stream function. 


\section{Acknowledgements}

Some of this work was carried out during the course of my participation in the Turbulence Workshop at the Kavli Institute for Theoretical Physics, Santa Barbara. I am thankful to Professors Katepalli Sreenivasan, Grisha Falkovich and Eberhard Bodenschatz for the invitation. I am thankful to Professors Grisha Falkovich and Jens Juul Rasmussen for the valuable remarks and suggestions. I am thankful to the referees for the valuable remarks and helpful criticism. This research was supported in part by NSF grant No. PHY05-51164.

\section{References}

[1] J.D. Gibbon: Physica D 237, 1894, (2008).

[2] E. Weidemann, Nonlin. Anal. 28, 727, (2011).

[3] P. Constantin: SIAM Rev. 36, 73, (1994).

[4] J. Leray: Acta Math. 63, 193, (1934).

[5] J. T. Beale, T. Kato and A. Majda: Comm. Math. Phys. 94, 61, (1989).

[6] A. Majda: SIAM Rev., 33, 349, (1991).

[7] J. Jucha, H. Xu, A. Pumir and E. Bodenschatz: Phys. Rev. Lett. 113, 054501, (2014).

[8] P. Constantin and C. Fefferman: Indiana Univ. Math. J. 42, 775, (1993).

[9] P. Constantin, C. Fefferman and A. Majda: Commun Partial Diff. Eqns. 21, 559, (1996).

[10] V. P. Ruban, D. I . Podolsky and J. J. Rasmussen: Phys. Rev. E 63, 056306, (2001).

[11] D. W. Moore: Proc. Roy. Soc. (London) A 365, 105, (1979).

[12] M. E. Brachet, M. Meneguzzi, A. Vincent, H. Politano and P.-L. Sulem: Phys. Fluids A 4, 2845, (1992).

[13] A. Majda and A. Bertozzi: Vorticity and Incompressible Flow, Ch. 5, Cambridge University Press, (2002).

[14] U. Frisch: Turbulence - The Legacy of A. N. Kolmogorov, Cambridge University Press, (1995).

[15] A. Pumir and E. Siggia: Phys. Fluids 2, 220, (1990).

[16] R. B. Pelz: Phys. Rev. E 55, 1617, (1997).

[17] R. Grauer, C. Marliani and K. Germaschewski: Phys. Rev. Lett. 80, 4177, (1998).

[18] G. Falkovich, K. Zawadezki and M. Vergassola: Rev. Mod. Phys. 73, 913, (2001).

[19] B. Mandelbrot: in Turbulence and Navier - Stokes Equations, Ed. R. Temam, Lecture Notes on Mathematics, Vol. 565, Springer-Verlag, (1975). 
[20] U. Frisch, P.-L. Sulem and M. Nelkin: J. Fluid Mech. 87, 719, (1978).

[21] G. Paladin and A. Vulpiani: Phys. Rev. A 35, 1971, (1987).

[22] K. R. Sreenivasan and C. Meneveau: Phys. Rev. A 38, 6287, (1988).

[23] A. A. Mailybaev: Phys. Rev. E 87, 053011, (2013).

[24] B. K. Shivamoggi: Physica A 390, 1534, (2011).

[25] B. K. Shivamoggi: Ann. Phys. 243, 177, (1995) and 318, 497, (2005).

[26] A. A. Mailybaev: Phys. Rev. E 85, 066317, (2012).

[27] B. B. Kadomtsev and V. I. Petviashvili: Sov. Phys. Dokl. 18, 115, (1973).

[28] P. Tabeling: Phys. Rep. 362, 1, (2002).

[29] G. Eyink: Nonlinearity 14, 787, (2001).

[30] M. C. Lopes Filho, A. L. Mazzucato and H. J. Nussenzveig: Arch. Rational Mech. Anal. 179, 353, (2006).

[31] J. Paret, M. C. Jullien and P. Tabeling: Phys. Rev. Lett. 83, 3418, (1999).

[32] K. Schneider and M. Farge: Phys. Rev. Lett. 95, 244502, (2005).

[33] J. C. McWilliams: J. Fluid Mech. 146, 21, (1984).

[34] Z. She, E. Jackson and S. A. Orszag: Nature 344, 226, (1990).

[35] B. K. Shivamoggi: Ann. Phys. 323, 444, (2008).

[36] B. K. Shivamoggi: arXiv 1209. 3311, (2014).

[37] M. C. Jullien: Phys. Fluids 15, 2228, (2003).

[38] S. Kida: J. Phys. Soc. Japan 54, 2840, (1985).

[39] E. A. Kuznetsov, V. Naulin, A. H. Nielsen and J. Juul Rasmussen: Phys. Fluids 19, 105110, (2007).

[40] B. K. Shivamoggi, G. J. F. van Heijst and J. Juul Rasmussen: Phys. Lett. A 374, 2309, (2010).

[41] B. K. Shivamoggi: J. Phys. A: Math. Gen. 23, 1689, (1990).

[42] H. A. Rose and P. L. Sulem: J. de Phys. T39, 441, (1978).

[43] J. Charney: Publ. Kosjones Nor. Vidensk. Akad. Oslo 17, 3, (1948).

[44] J.-M Hoyer and R. Sadourny: J. Atmos. Sci. 39, 707, (1982).

[45] P. Constantin, Q. Nie and N. Schorghofer: Phys. Lett. A 241, 168, (1998).

[46] K. Ohkitani and M. Yamada: Phys. Fluids 9, 876, (1997).

[47] R. K. Scott and D. G. Dritschel: Phys. Rev. Lett. 112, 144505, (2014). 
[48] V. Carbone and A. Pouquet: Springer Lect. Notes Phys. 778, 71, (2009).

[49] G. K. Batchelor: Proc. Roy. Soc. (London) A 201, 405, (1950).

[50] S. Kida, S. Yanase and J. Mizushima: Phys. Fluids A 3, 457, (1991).

[51] A. Bershadskii and K. R. Sreenivasan: Phys Rev. Lett. 93, 064501, (2004).

[52] B. K. Shivamoggi: Ann. Phys. 253, 239, (1997). Erratum in Ann. Phys. 312, 270, (2004).

[53] P. S. Iroshnikov: Sov. Astron. 7, 566, (1964).

[54] R. H. Kraichnan: Phys. Fluids 8, 1385, (1965).

[55] S. A. Orszag and C. M. Tang: J. Fluid Mech. 90, 129, (1979). 